\title{
Second-Order Occlusion-Aware Volumetric Radiance Caching
}

\author{
Julio Marco* \\ Universidad de Zaragoza, I3A \\ Wojciech Jarosz \\ Dartmouth College
}

\author{
Adrian Jarabo \\ Universidad de Zaragoza, I3A \\ Diego Gutierrez \\ Universidad de Zaragoza, I3A
}

\begin{abstract}
Accurate simulation of light transport in participating media is expensive, due to the many scattering events. However, the bandlimiting effect of scattering in media makes this kind of light transport very suitable for adaptive sampling and reconstruction techniques. In this work we present a novel algorithm that adaptively samples radiance from sparse points in the medium using up-to second-order occlusion-aware derivatives to determine when interpolation is appropriate. We derive our metric from each point's incoming light field. We use a proxy triangulation-based representation of the radiance reflected by the surrounding medium and geometry to efficiently compute the first- and second-order derivatives of the radiance at the cache points while accounting for occlusion changes. We validate the quality of our approach on a self-contained two-dimensional model for light transport in media. Then we show how our results generalize to practical threedimensional scenarios, where we show much better results while reducing computation time up to a $30 \%$ compared to previous work.
\end{abstract}

\section{CCS CONCEPTS}

\section{-Computing methodologies $\rightarrow$ Ray tracing;}

\section{KEYWORDS}

radiance caching, participating media, radiance derivatives

\section{ACM Reference format:}

Julio Marco, Adrian Jarabo, Wojciech Jarosz, and Diego Gutierrez. 2017. Second-Order Occlusion-Aware Volumetric Radiance Caching. In Proceedings of SIGGRAPH '17 Posters, Los Angeles, CA, USA, fuly 30 - August 03, 2017, 2 pages.

DOI: $10.1145 / 3102163.3102242$

\section{INTRODUCTION}

Accurate simulation of the complex lighting effects produced by participating media in the presence of arbitrary geometry remains a challenging task. Monte Carlo-based methods like path tracing numerically approximate the radiative transfer equation (RTE) [Chandrasekhar 1960], but they lack memory and are largely blind to the radiance signal, which leads to redundant computations and high cost. A common strategy to increase efficiency is to adaptively sample radiance based on its frequency content, limiting

\footnotetext{
${ }^{*}$ Corresponding author: juliom@unizar.es

Permission to make digital or hard copies of part or all of this work for personal or classroom use is granted without fee provided that copies are not made or distributed for profit or commercial advantage and that copies bear this notice and the full citation on the first page. Copyrights for third-party components of this work must be honored. For all other uses, contact the owner/author(s).

SIGGRAPH '17 Posters, Los Angeles, CA, USA

(c) 2017 Copyright held by the owner/author(s). 978-1-4503-5015-0/17/07 ..\$15.00 DOI: $10.1145 / 3102163.3102242$
}

the sampling density in regions where radiance barely changes, and placing more samples in regions with higher frequency variation [Zwicker et al. 2015]. Based on this principle, volumetric radiance caching [Jarosz et al. 2008] computes and stores radiance at sparse cache points in the medium, and uses these samples to reconstruct radiance at nearby locations whenever possible. The method is based on first-order translational derivatives of the radiance, which are used to i) determine how far away a cache point can be reused while controlling error, and ii) improve reconstruction quality by extrapolating the cached radiance values along their gradients. Unfortunately, this method ignores visibility changes and higher order derivatives, leading to suboptimal cache point distributions which fail to properly sample high-frequency features such as occlusions, while simultaneously oversampling other regions of the scene. This results in reduced efficiency and visible rendering artifacts. Additionally, second-order illumination derivatives have proven to be a powerful and principled tool for sparsely sampling and interpolating surface irradiance [Jarosz et al. 2012; Schwarzhaupt et al. 2012].

\section{OUR APPROACH}

Inspired by these recent developments, we propose a new secondorder, occlusion-aware radiance caching method for participating media which overcomes the limitations of current state-of-theart methods. To this end, we develop a Hessian-based metric for controlling the error introduced by first-order extrapolation of media radiance, and introduce a novel approach to compute firstand second-order occlusion-aware derivatives of both single and multiple scattering. In addition, we extend recent work on 2D radiometry, currently limited to surfaces [Jarosz et al. 2012], and derive a $2 \mathrm{D}$ theory of light transport in participating media. We use this framework to illustrate and analyze the limitations of the state of the art, as well as the benefits of our proposed method. We demonstrate the generality of our approach by deriving occlusionaware derivatives of 3D media radiance and applying our Hessianbased metric to 3D cache distributions, showing that the benefits predicted by our $2 \mathrm{D}$ analysis hold equally in $3 \mathrm{D}$.

Second-order error control. The error in radiance caching is controlled by a tolerance value $\varepsilon$, and depends both on how radiance is extrapolated, and on the radiance moments at cache point $\mathbf{x}$. These define a valid bounding region $\boldsymbol{N}$ where a point $\mathbf{x}^{\prime}$ can be used for extrapolation. Using a second-order Taylor expansion of media radiance as an oracle of the actual radiance, we can bound the error of first-order radiance interpolation for both $2 \mathrm{D}$ and 3D media as

$$
R_{2 \mathrm{D}}^{\lambda_{i}}=\sqrt[4]{\frac{4 L(\mathbf{x}) \varepsilon}{\pi\left|\lambda_{i}\right|}}, \quad R_{3 \mathrm{D}}^{\lambda_{i}}=\sqrt[5]{\frac{15 L(\mathbf{x}) \varepsilon}{4 \pi\left|\lambda_{i}\right|}}
$$



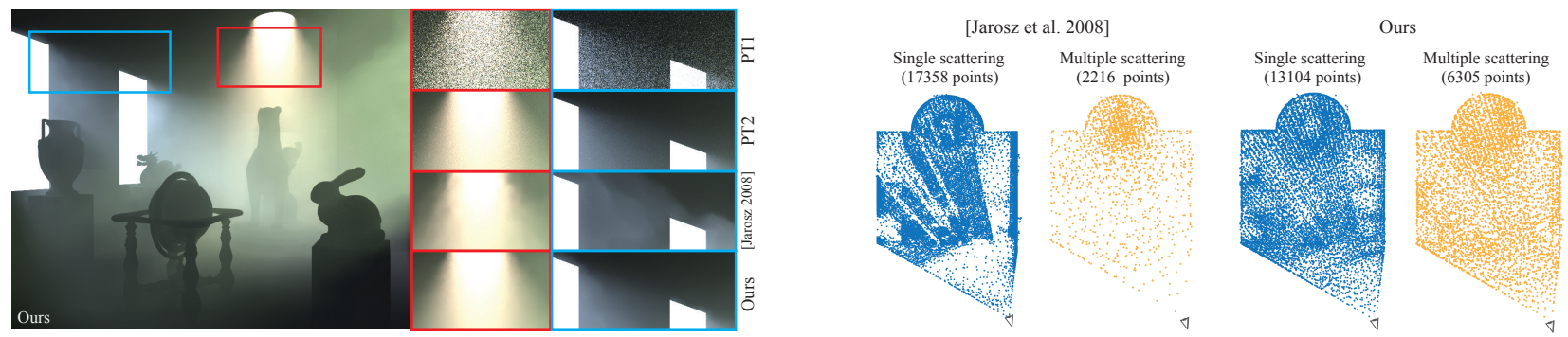

Figure 1: PT1: Path tracing, 2k samples/ray, 2h. PT2: Path tracing, 500k samples/ray, 500h. [Jarosz et al. 2008]: Occlusionunaware, gradient-based error metric, $\sim 19 \mathrm{k}$ cache points, 16k samples/cache, 155 minutes. Ours: Occlusion-aware, Hessianbased metric, $\sim 19 \mathrm{k}$ cache points, 16k samples/cache, 154 minutes. Right: Cached point distributions comparison as seen from above for both single and multiple scattering.

where $\lambda_{i}$ is the $i$-th eigenvalue of the radiance Hessian. These allow us to bound valid regions as ellipses (in 2D) or ellipsoids (in 3D), efficiently sampling the media with cache points depending on the radiance curvature. Only points that ensure a maximum error $\hat{\epsilon}^{\prime}<\varepsilon$ are used to interpolate radiance at $\mathbf{x}^{\prime}$, and therefore the error of the weighted sum is equally upper-bounded by $\varepsilon$.

Computing occlusion-aware media derivatives. In the spirit of Schwarzhaupt and colleagues surface approach [Schwarzhaupt et al. 2012], we transform radiance integration around media points $\mathbf{x}$ into a discrete set of virtual piecewise linear representations of the geometry and media. These allow us to represent incoming radiance as an occlusion-free triangulation (see a 2D example in Figure 2) where occlusions result in slanted triangles (red). Single scattering triangulation is computed at surface level, while multiple scattering is computed with triangulations at ray-marched locations. Under a sufficiently fine subdivision, we can express the contribution of every triangle $\ell_{j}$ to the outgoing light field at $\mathbf{x}$ as

$$
L_{j}\left(\mathbf{x} \rightarrow \vec{\omega}_{o}\right)=f\left(\mathbf{x}, \vec{\omega}_{i}, \vec{\omega}_{o}\right) T_{r}\left(\mathbf{x}, \ell_{j}\right) L_{o}\left(\ell_{j} \rightarrow \vec{\omega}_{i}\right) F_{\ell_{j}}(\mathbf{x}),
$$

with $f, T_{r}$ and $F_{\ell_{j}}$ being the phase function, transmittance, and form factor of the triangle $\ell_{j}$ respectively. By differentiating Equation (2) with respect to $\mathbf{x}$, we can approximate outgoing radiance and its derivatives at $\mathbf{x}$ as a summation over all segments $\ell_{j} \in \mathcal{L}_{i}$ and concentric rings $r_{i} \in \mathcal{R}$ including the surface subdivision,

$$
\begin{aligned}
L\left(\mathbf{x} \rightarrow \vec{\omega}_{o}\right) & \approx \sum_{r_{i} \in \mathcal{R}} \sum_{\ell_{j} \in \mathcal{L}_{i}} \frac{L_{j}\left(\mathbf{x} \rightarrow \vec{\omega}_{o}\right)}{\operatorname{pdf}\left(r_{i}\right)}, \\
\nabla L\left(\mathbf{x} \rightarrow \vec{\omega}_{o}\right) & \approx \sum_{r_{i} \in \mathcal{R}} \sum_{\ell_{j} \in \mathcal{L}_{i}} \frac{\nabla L_{j}\left(\mathbf{x} \rightarrow \vec{\omega}_{o}\right)}{\operatorname{pdf}\left(r_{i}\right)} \\
\mathbf{H} L\left(\mathbf{x} \rightarrow \vec{\omega}_{o}\right) & \approx \sum_{r_{i} \in \mathcal{R}} \sum_{\ell_{j} \in \mathcal{L}_{i}} \frac{\mathbf{H} L_{j}\left(\mathbf{x} \rightarrow \vec{\omega}_{o}\right)}{\operatorname{pdf}\left(r_{i}\right)} .
\end{aligned}
$$

Differentiating Equation (2) requires computing gradients and Hessians of transmittance, phase function and triangle-media form factors, and applying the differentiation product rule. These expressions present closed-form solutions for homogeneous media and phase functions such as Henyey-Greenstein, allowing for efficient computation of media derviatives.

We demonstrate the use of our approach in Figure 1 where we compare our results against Jarosz and colleagues gradient-based occlusion-unaware method [Jarosz et al. 2008] and path tracing.

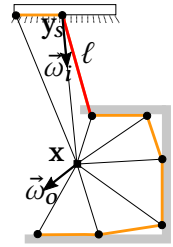

(a)

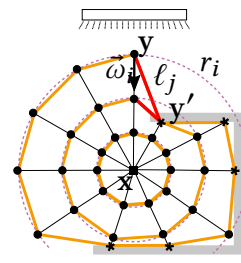

(b)

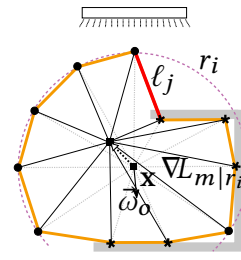

Figure 2: We construct subdivisions of the scene surfaces and media as seen from cached media locations $\mathrm{x}$ for (a) single and (b) multiple scattering, and provide closed-form expressions for occlusion-aware media radiance derivatives.

Note how our method converges to the correct solution, while path tracing methods are prohibitively expensive, and occlusionunaware radiance caching fails to capture high frequency changes around the windows or in the hole in the ceiling. Point distributions on Figure 1, right, show how the gradient-based method unevenly places most of the cache points near walls and the floor, while our method correctly samples high frequency regions with more points near the light shafts boundaries through the windows.

\section{ACKNOWLEDGEMENTS}

This research has been funded by by DARPA (project REVEAL), an ERC Consolidator Grant (project CHAMELEON), the Spanish Ministry of Economy and Competitiveness (projects TIN2016-78753$\mathrm{P}$ and TIN2016-79710-P), and the Gobierno de Aragón.

\section{REFERENCES}

Subrahmanyan Chandrasekhar. 1960. Radiative Transfer. Dover.

Wojciech Jarosz, Craig Donner, Matthias Zwicker, and Henrik Wann Jensen. 2008. Radiance Caching for Participating Media. ACM Transactions on Graphics (Presented at SIGGRAPH) 27, 1 (March 2008), 7:1fi??7:11. DOI : https://doi.org/10.1145/1330511. 1330518

Wojciech Jarosz, Volker Schnefeld, Leif Kobbelt, and Henrik Wann Jensen. 2012. Theory, Analysis and Applications of 2D Global Illumination. ACM Transactions on Graphics (Presented at SIGGRAPH) 31, 5 (Sept. 2012), 125:1fi??125:21. DOI : https://doi.org/10. $1145 / 2231816.2231823$

Jorge Schwarzhaupt, Henrik Wann Jensen, and Wojciech Jarosz. 2012. Practical HessianBased Error Control for Irradiance Caching. ACM Transactions on Graphics (Proceedings of SIGGRAPH Asia) 31, 6 (Nov. 2012). DOI : https://doi.org/10.1145/2366145. 2366212

Matthias Zwicker, Wojciech Jarosz, Jaakko Lehtinen, Bochang Moon, Ravi Ramamoorthi, Fabrice Rousselle, Pradeep Sen, Cyril Soler, and Sung-Eui Yoon. 2015. Recent Advances in Adaptive Sampling and Reconstruction for Monte Carlo Rendering. Computer Graphics Forum (Proceedings of Eurographics) 34, 2 (May 2015), 667-681. DOI : https://doi.org/10.1111/cgf.12592 